\title{
Zukunftsgestalter
}

Silvia Czerwinski* und Tatyana Tasche

\section{Aufbau und Konzeption von Selbstlernkursen zu digitalen Kompetenzen}

https://doi.org/10.1515/bfp-2020-0102

Zusammenfassung: In einem vierjährigen Projekt hat die Universität Göttingen Online-Kurse entwickelt, die Studierende bei der Entwicklung von digitalen Kompetenzen unterstützen sollen. Die Kurse stehen den Studierenden als Selbstlernmaterialien über das universitäre Lernmanagementsystem Stud.IP/ILIAS zur Verfügung. Für Nichtangehörige der Universität besteht ein freier Zugang über die Plattform OpenILIAS. Da die Kurse unter einer CreativCommons-Lizenz veröffentlicht wurden, können sie in Teilen oder im Ganzen weiterverbreitet, verändert und ergänzt werden.

Schlüsselwörter: Digitale Kompetenzen; E-Learning; OER

\section{Conception and Design of Online Courses on Digital Skills}

Abstract: In a four-year project, the University of Göttingen has developed online courses to support students in developing digital skills. The courses are available to students as self-study materials via the university's learning management system Stud.IP/ILIAS. Non-members of the university have free access via the platform OpenILIAS. As the courses are published under a Creative Commons licence, they can be redistributed, modified, and supplemented in part or in whole.

Keywords: Digital skills; e-learning; OER

\section{Ziel des Projekts „Digitale Kompetenzen“}

Die bundesweite Einführung von weitgehend digitalen Semestern aufgrund der Covid-19-Pandemie zeigt eindringlich, wie wichtig das Erlernen eines sicheren Umgangs mit digitalen Informationen und Werkzeugen für Studierende ist: Sie müssen über verschiedene Online-Kanäle

*Kontaktperson: Silvia Czerwinski, silvia.czerwinski@tib.eu

Tatyana Tasche, tatyana.tasche@uni-goettingen.de kommunizieren und in kollaborativen Teams Daten- oder Multimedia-Projekte bearbeiten. Dafür finden und bewerten die Studierenden Informationen in digitalen Publikationen und Suchräumen. Zudem visualisieren und präsentieren sie ihre Ergebnisse mit unterschiedlichen Methoden auf verschiedenen Online-Plattformen. Kurz: Der Alltag der Studierenden ist durchweg digital geprägt.

Um sich souverän in digitalen Umgebungen bewegen zu können, werden bestimmte Kenntnisse und Fähigkeiten benötigt, die Studierenden oft erst erlernen müssen. Dies gilt insbesondere für die Studierenden der Geisteswissenschaften, da hier die Arbeit mit Daten sowie mit digitalen Methoden und Werkzeugen immer größeren Raum einnimmt. Die Universität Göttingen entwickelt deshalb in einem vierjährigen Projekt Online-Lernangebote, die die Studierenden bei dem Erwerb bzw. dem Ausbau ihrer digitalen Kompetenzen unterstützen sollen.

Das Projekt „Digitale Kompetenzen für Studierende“ (DigKom) ${ }^{1}$ hat eine Laufzeit von vier Jahren (2016-2020) und ist Teil der zweiten Förderungsphase des übergreifenden Projektes Göttingen Campus Q ${ }^{\text {PLUS }}{ }^{2}$ Mit über 25 Einzelmaßnahmen sollen darin die Studienbedingungen verbessert und die Qualität in der Lehre erhöht werden. Mit dem Projekt Campus Q ${ }^{\text {PLUS }}$ ist die Universität Göttingen seit 2011 am Qualitätspakt Lehre beteiligt, der von Bund und Ländern finanziert wird. Das Teil-Projekt DigKom wird gemeinsam von der Abteilung Studium und Lehre (Bereich Digitales Lernen und Lehren) und der Niedersächsischen Staatsund Universitätsbibliothek Göttingen (Benutzungsabteilung und eResearch Alliance ${ }^{3}$ ) durchgeführt. In dem Projekt sind zwei Stellen angesiedelt: 0,75 VZÄ Digital Humanist und 0,75 VZÄ Instructional Designer. Über zentrale Studienqualitätsmittel werden zusätzlich drei studentische Hilfskräfte finanziert.

Ziel des Projektes DigKom ist die Bereitstellung von Selbstlernkursen, die in enger Abstimmung mit den Lehrenden und den jeweiligen Experten des Bereichs ent-

1 https://www.uni-goettingen.de/de/629608.html.

2 https://www.uni-goettingen.de/de/217934.html.

$3 \mathrm{http} / / /$ www.eresearch.uni-goettingen.de. 
wickelt werden. Die Lerninhalte werden über Videos, kurze Texte, Infografiken und vielen Quiz als Module im Lernmanagementsystem Stud.IP/ILIAS vermittelt. Diese Module können in die Lehre eingebunden werden und stehen den Studierenden als zusätzliche, freie Lernmaterialien zur Verfügung. Über eine Kopie in OpenILIAS ${ }^{4}$ sind die Lernangebote zudem als Open Educational Resources (OER) nachnutzbar.

$\mathrm{Zu}$ Beginn des Projektes erfolgte eine Bestimmung des Feldes „digitale Kompetenzen“. Dabei wurde auf bereits vorliegende Kompetenzmodelle zurückgegriffen. Um herauszufinden, inwiefern die Studierenden der Geisteswissenschaften der Universität Göttingen bereits über diese Kompetenzen verfügen bzw. wie deren eigene Einschätzung dazu ist, wurde im SoSe 2017 eine Onlineumfrage unter den Studierenden durchgeführt. Aufgrund der Ergebnisse der Umfrage wurden relevante Themen für Lernangebote identifiziert und bis Projektende insgesamt fünf deutschsprachige Selbstlernkurse erstellt und drei der Kurse ins Englische übersetzt.

\section{Was sind digitale Kompetenzen?}

Nach einer ersten Recherche zum Thema „digitale Kompetenzen" konnten die vier wichtigsten Handlungsfelder identifiziert werden: Kommunikation, Medien, Daten und Informatik. Je nachdem, welches Feld näher betrachtet wird, ergibt sich eine andere Definition von „digitalen Kompetenzen" und dementsprechend eine andere Ausrichtung der Lernangebote.

Betrachtet man die bereits bestehenden Angebote von Universitäten, legen auffällig viele den Schwerpunkt auf den Umgang mit digitalen Medien bzw. auf die Kommunikation im Internet, z. B. die frei zugänglichen Online-Kurse „Digital Literacy“5 der Fachhochschule Lübeck oder „Digital Footprint" ${ }^{6}$ der University of Edinburgh. Andere Angebote erweitern den von Bibliotheken seit langem bespielten Bereich der Informationskompetenz lediglich um digitale Begriffe wie z.B. der Online-Kurs „Information \& Digital Literacy for University Success ${ }^{\text {“7 }}$ der University of Sydney.

Ein weiteres Kompetenz-Feld ist das der „Digitalen Bildung“. Hier stehen vorrangig die Kompetenzen der Lehrenden bzw. der Lehramtsstudierenden beim Einsatz von

$4 \mathrm{http}$ //openilias.uni-goettingen.de/openilias/goto_openilias_cat_ 368.html.

5 https://www.oncampus.de/integration/digital-literacy.

6 https://www.coursera.org/learn/digital-footprint.

7 https://www.coursera.org/learn/digital-literacy.
E-Learning im Fokus, wie sie z.B. im Zertifikatsprogramm „Lernen und Lehren in der digitale Gesellschaft" ${ }^{\text {“8 }}$ der Universität Trier vermittelt werden. Ziel ist es dabei, digitale Medien kompetent im Unterricht einzubinden und die Lernenden im Umgang damit zu schulen, wie es z.B. im „Monitor Digitale Bildung“9 der Bertelsmann Stiftung ausführlich untersucht wurde und vom digitalen Bildungspakt ${ }^{10}$ unterstützt wird.

Im Rahmen der digitalen Bildung wird noch ein anderer Punkt diskutiert, nämlich wie früh und wie intensiv Informatik ein Schulfach sein soll. Für die curriculare Verankerung der Informatik bereits in der Grundschule sowie informatischer Inhalte in anderen Fächern argumentieren z. B. Informatikdidaktikerinnen wie Ira Diethelm. ${ }^{11}$ Unterstützung erhält sie von der Gesellschaft für Informatik (GI), die in den Empfehlungen der Kompetenzen für informatische Bildung im Primarbereich ${ }^{12}$ sich dafür einsetzt, dass bereits Schulkinder Lösungen für Probleme in der digitalen Welt selbständig entwickeln können. Ein viel zitierter Ansatz bei dieser Art der digitalen Bildung ist das so genannte „Dagstuhl-Dreieck“: ${ }^{13}$ Die Lernenden nähern sich einem Problem oder einem Phänomen der digitalen Welt aus drei verschiedenen Richtungen, um ein ganzheitliches Bild zu erhalten und zu bestmöglichen Lösungen zu gelangen: Wie nutze ich das? Wie funktioniert das? Wie wirkt das?

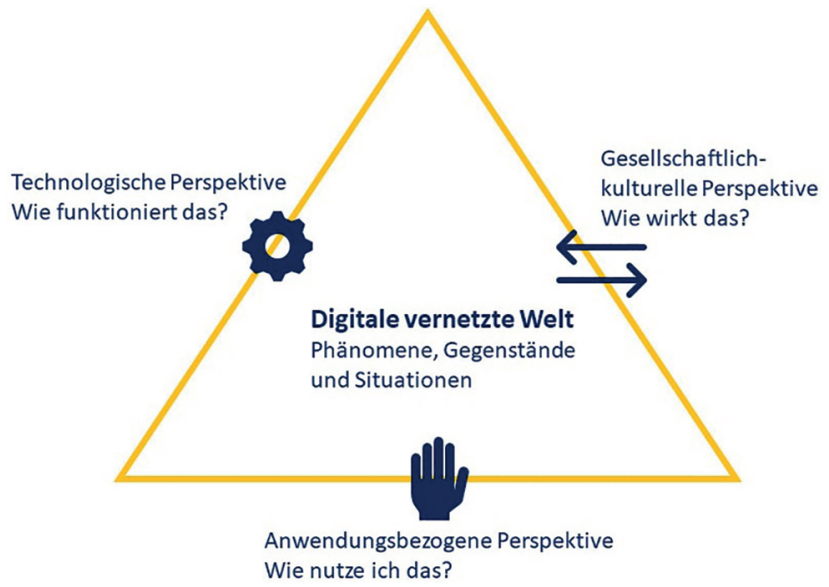

Abb. 1: Dagstuhl

8 https://www.uni-trier.de/index.php?id=64030.

9 https://www.bertelsmann-stiftung.de/de/publikationen/publikati on/did/monitor-digitale-bildung/.

$10 \mathrm{http}: / /$ digitaler-bildungspakt.de/.

11 https://www.bundestag.de/resource/blob/573972/37590b970d6d 530bcce7825efe39160e/Diethelm_Stellungnahme_37g-data.pdf.

12 https://gi.de/themen/beitrag/informatische-bildung-im-primarb ereich.

13 https://dagstuhl.gi.de/dagstuhl-erklaerung. 
In der Informatik ist der Fokus auf kreativen Lösungsansätzen grundlegend und wird als „Computational Thinking“ bezeichnet: Ein Problem wird in mehrere Punkte unterteilt und dann dafür eine schrittweise Lösung erarbeitet. Dieses algorithmische Denken steht am Anfang jedes Programmierenlernens und kann in andere Lebensbereiche übertragen werden. ${ }^{14}$ Man könnte diese Herangehensweise als unterste Ebene ansehen, auf die alle anderen digitalen Kompetenzen aufbauen.

Eine strukturierte Sammlung an digitalen Kompetenzbereichen wurde bereits 2016 von der Kultusministerkonferenz (KMK) erstellt.. ${ }^{15}$ Die Universität Göttingen (Bereich Digitales Lernen und Lehren) hat eine eigene Matrix zu digitalen Kompetenzen ${ }^{16}$ entwickelt und sie den Kompetenzfeldern der KMK zugeordnet. Auch bei der Entwicklung der Lernangebote im Projekt DigKom diente der Kompetenzrahmen der KMK als Referenzpunkt.

Eine eindeutige Abgrenzung von digitalen (Digital Literacy) und datenbezogenen Kompetenzen (Data Literacy) ist kaum zu leisten. Im Projekt DigKom werden diese Kompetenzen jedoch nur sehr rudimentär behandelt. Dies hat zwei Gründe: Zum einen bietet das Projekt „Daten Lesen Lernen “, ${ }^{17}$ federführend unter dem Zentrum für Statistik der Universität Göttingen, die Lehrveranstaltung „Data Literacy Basics“ für Studierende aller Fachrichtungen an. Zum anderen werden digitale Kompetenzen im Umgang mit Daten in den Geisteswissenschaften vorrangig über die datengetriebene Forschung in den Digital Humanities vermittelt. So wurden auch an der Universität Göttingen 2019 die bisherigen Bestrebungen im Institut für Digital Humanities ${ }^{18}$ gebündelt und ein Studiengang eingerichtet.

\section{Was benötigen die Studierenden?}

Nachdem die digitalen Kompetenzfelder im Projekt DigKom identifiziert waren, sollten sie mit den Bedarfen der Zielgruppe, also den Studierenden der Geisteswissenschaften der Universität Göttingen, abgeglichen werden. Dazu wurde 2017 eine Bedarfsanalyse durchgeführt. Zur Vorbereitung einer Online-Umfrage unter Studierenden wurden in Einzelgesprächen mit fünf Lehrenden der Universität Göttingen, die in ihrer Lehre bereits digitale Kom-

\footnotetext{
14 https://www.cs.cmu.edu/afs/cs/usr/wing/www/ct-german.pdf. 15 https://www.kmk.org/fileadmin/Dateien/veroeffentlichungen_b eschluesse/2018/Strategie_Bildung_in_der_digitalen_Welt_idF._vom _07.12.2017.pdf.

16 https://www.uni-goettingen.de/de/609498.html.

$17 \mathrm{https} / / /$ www.uni-goettingen.de/de/592287.html.

18 https://www.uni-goettingen.de/de/597374.html.
}

petenzen vermitteln, wichtige Themenbereiche festgelegt. Diese Themen wurden in einem Workshop mit Masterstudierenden und einer Vertreterin der Graduiertenschule für Geisteswissenschaften Göttingen (GSGG) diskutiert.

Aus den Ergebnissen des Workshops und der Einzelgespräche sowie anhand des Kompetenzrahmens der KMK wurden die endgültigen Fragen für eine Online-Umfrage unter Studierenden der Geisteswissenschaften entwickelt. In einer Vollerhebung unter Studierenden der Philosophischen und der Theologischen Fakultäten der Universität Göttingen im SoSe 2017 sollte demnach herausgefunden werden, welche digitalen Kompetenzen bei den Studierenden bereits vorhanden sind und welche konkrete digitale Kompetenzen ihrer Meinung nach für das Studium und das spätere Berufsleben benötigt werden. Daraus ergaben sich priorisierte Arbeitsbereiche, in denen neue Lernangebote entwickelt werden.

Die gesamte Umfrage gliederte sich in neun Themenbereiche (plus der Abfrage demografischer Daten), in denen insgesamt 22 Fragen gestellt wurden. Bis auf drei Freitextfragen wurden hauptsächlich Multiple- bzw. SingleChoice-Frageformen verwendet. Als Umfragetool wurde die Software SoSci Survey über die Hochschullizenz der Wirtschaftswissenschaftlichen Fakultät verwendet. Den Aufruf und den Zugangslink zur Umfrage bekamen die Studierenden über eine Mail der Dekanate der Philosophischen bzw. Theologischen Fakultät. Es wurden insgesamt 5401 Studierende angeschrieben, davon 5027 aus der Philosophischen und 374 aus der Theologischen Fakultät. 294 Personen haben die Umfrage begonnen und 205 Personen haben sie bis zur letzten Frage beantwortet. Dies ergibt eine Rücklaufquote von 5,44 \% bzw. 3,79 \%.

Bei der Auswertung der Umfrage stellte sich zum einen heraus, dass die bis dahin bereits im Rahmen des Projekts entwickelten bzw. sich in der Entwicklung befindenden Lernangebote den Bedarfen der Studierenden entsprechen. Dies betraf insbesondere die Kurse zu Open Educational Resources (OER) sowie zur Literaturrecherche. Zum anderen formulierten die befragten Studierenden, dass die Arbeit mit Daten für ihr Studium bzw. ihr späteres Berufsleben sehr relevant sei, ohne dass sie jedoch den abgefragten Arbeitsschritten eine sinnvolle Bedeutung geben können. Im Ganzen wurden Aspekte zur Kommunikation, Arbeit im Team, Recherche/Informationsbeschaffung, Präsentation/Publikation, Daten (inkl. Datensicherung) und Informationsverarbeitung abgefragt.

Neben den inhaltlichen Aspekten wurden auch Fragen zur Motivation gestellt. Da im Projekt DigKom reine Selbstlernkurse entwickelt werden, war es von großem Interesse $\mathrm{zu}$ erfahren, warum die Studierenden einen Kurs bis zum Ende durchführen bzw. was sie evtl. zum Abbruch veran- 


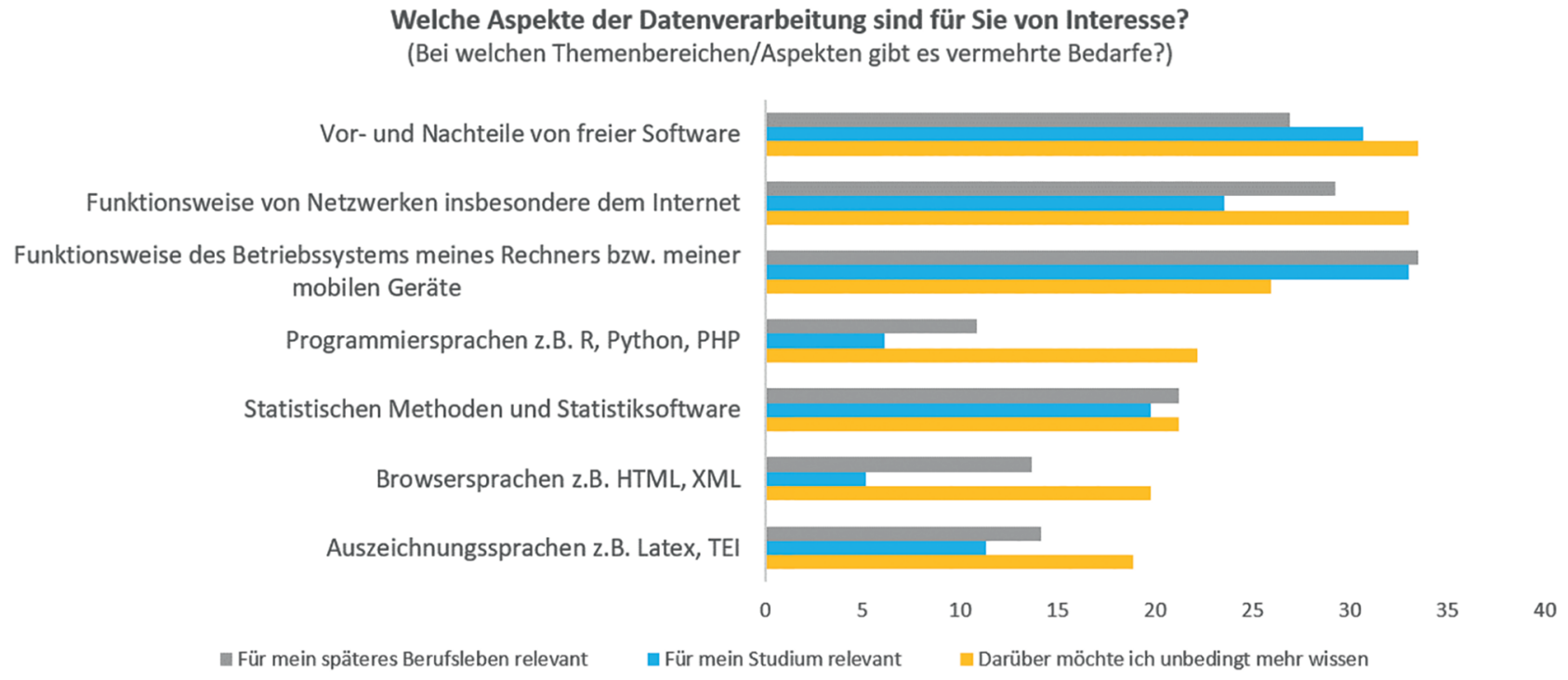

Abb. 2: Interesse

Welche Aspekte motivieren Sie am ehesten, einen Online-Selbstlernkurs erfolgreich durchzuführen?

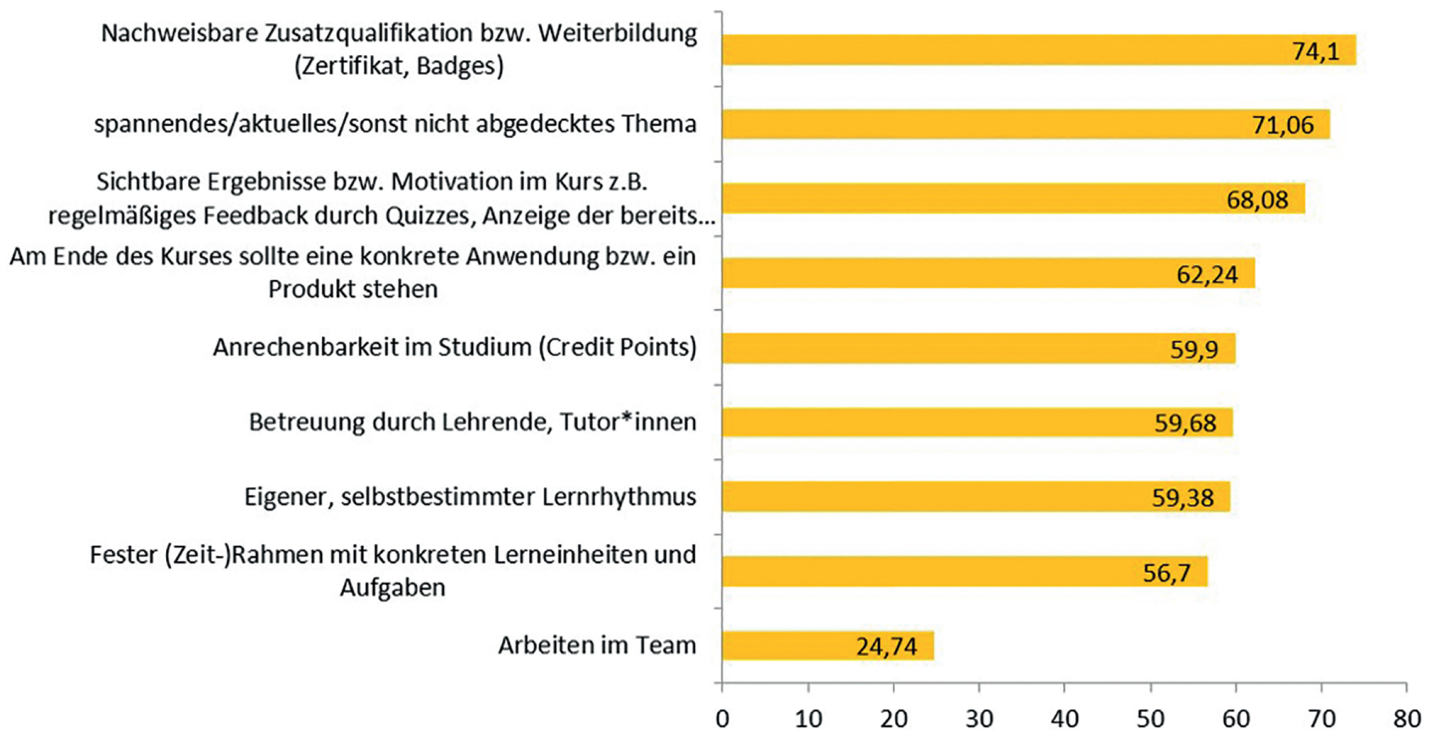

Abb. 3: Motivation

lassen würde. Von Konzepten des Blended Learning oder einer Begleitung über ein Forum oder Wiki wurde abgesehen, weil innerhalb des Projekts der Fokus auf das Erstellen der Kurse gelegt wurde. Eine didaktische Begleitung der Lernenden erfolgt lediglich in solchen Kursen, die von Lehrenden in ihre Veranstaltungen übernommen wurden. Dies gilt z. B. für den OER-Kurs bei den Lehramtsstudierenden oder den Literaturrecherchekurs in der Englischen Philologie und der Interkulturellen Germanistik.
Die Studierenden erhalten demnach nur in ausgewählten Seminaren ECTS-Punkte. In allen Kursen ist es allerdings möglich, eine Teilnahmebescheinigung zu erhalten, wie es laut Umfrage von den Studierenden gewünscht ist. 


\section{Erstellung von ILIAS-Lernmodulen}

Die Selbstlernkurse werden im Projekt DigKom in Anlehnung an das ADDIE-Modell aus dem Instructional Design ${ }^{19}$ entwickelt. Dieses gliedert sich in fünf Phasen:

1. In der Analysephase wird das Thema erfasst und Experten dazu auf dem Campus identifiziert.

2. In der Designphase werden die groben Lernziele und eine erste inhaltliche Struktur des Kurses erstellt. Diese werden in einem mehrstündigen Workshop mit Experten auf dem Feld, die möglichst unterschiedliche Aspekte abdecken, diskutiert und konkretisiert.

3. In der Entwicklungsphase (engl. Development) wird nach bereits vorhandenen Materialien unter freien Lizenzen recherchiert. Diese werden im nächsten Schritt evtl. bearbeitet, z. B. werden Videos geschnitten oder mit deutschen Untertiteln versehen, oder Grafiken werden angepasst. Außerdem werden in dieser Phase neue Materialien erstellt. Dies sind hauptsächlich Texte, erklärende Grafiken bzw. Infografiken und verschiedene H5P-Quiz.

4. In der Implemetierungsphase werden die verschiedenen Materialien im Lernmanagementsystem - an der Universität Göttingen wird hauptsächlich Stud.IP/ ILIAS eingesetzt - eingebunden und angepasst. Außerdem werden zu dem eigentlichen Lernmodul weitere Module wie ein Abschlusstest, ein Glossar oder eine Evaluation angelegt.

5. In der Evaluierungsphase wird der Kurs zunächst inhaltlich bzw. didaktisch von den Experten getestet. Außerdem werden technische Tests auf verschiedenen Geräten und Systemen durchgeführt. Schlussendlich wird ein Feedback der Zielgruppe eingeholt. Zunächst werden ausgewählte Studierende gebeten, ein ausführliches Feedback zu erstellen. Sobald der Kurs für alle online zugänglich ist, kann von allen Teilnehmenden ein Evaluationsbogen ausgefüllt und eine Bewertung abgegeben werden. Die Möglichkeit des Evaluationsbogens wird eher spärlich genutzt. Insbesondere die Angaben in dem darin enthaltenen Freitextfeld liefern allerdings hilfreiche Anmerkungen und Ideen, die möglichst zeitnah umgesetzt werden.

Mit diesem Workflow wurden im Projekt DigKom fünf Kurse in deutscher Sprache erstellt. Drei davon wurden ins Englische übersetzt und für die Gruppe der internationalen Studierenden leicht angepasst. Die Kurse richten sich an

19 https://www.e-teaching.org/didaktik/theorie/instruktionsdesign /instruktionsdesign.pdf.
Anfänger bzw. leicht Fortgeschrittene und bestehen aus 4-6 Kapiteln mit einer durchschnittlichen Länge von ca. 20-30 Minuten. Aufgrund des modularen Aufbaus bleibt der Lernvorgang flexibel, d.h., die Lernenden bestimmen ihr eigenes Lerntempo, den Ablauf des Kurses und die Inhaltstiefe. Sie lernen demnach nur das, was sie entsprechend ihrer individuellen Erfordernisse brauchen. Es wurden folgende Kurse entwickelt:

Freie Bildungsmaterialien: Open Educational Resources (OER) leicht gemacht.

OER sind Lehr- und Lernmaterialien, die kostenfrei genutzt, bearbeitet und weitergegeben werden dürfen. Der Kurs soll einen ersten praktischen Einstieg in OER bieten und die Studierenden für ein komplexes Thema insbesondere für urheberrechtliche Fragen und Verwendung von freien Lizenzen sensibilisieren.

Literaturrecherche: Wie Sie finden, was Sie suchen. (auch in Englisch)

In diesem Kurs lernen die Studierenden effektive Suchstrategien für die Recherche in Fachdatenbanken und Bibliothekskatalogen kennen. Außerdem erhalten sie wertvolle Tipps für die Beurteilung der wissenschaftlichen Qualität der gefundenen Informationen.

Informationsvisualisierung: Daten auf den Punkt gebracht. (auch in Englisch)

In diesem Kurs lernen die Studierenden unterschiedliche Diagrammarten und Visualisierungsformen kennen und erfahren, welche Grafiken oder andere Formen der Visualisierung für welche Daten geeignet sind. Außerdem erhalten sie Tipps für ein gutes Design, um ihre Daten und Informationen bestmöglich darzustellen.

Informationstechnologie: IT so leicht wie nie. (auch in Englisch)

Nach Abschluss dieses Kurses sind die Studierenden mit den grundsätzlichen Prinzipien und Prozessen der Informationstechnologie vertraut und in der Lage, sich in vertiefende bzw. fachspezifische Methoden und Werkzeuge ihres Studiums einzuarbeiten.

Datensicherheit: Nehmen Sie Ihre digitalen Fußspuren in die Hand.

Mit diesem Kurs können die Studierenden abschätzen, welche Daten sie täglich preisgeben und welche Auswirkungen dies hat. Außerdem sind sie in der Lage, Maßnahmen zum Schutz ihrer Daten zu ergreifen.

Alle Kurse sind für die Studierenden in ihrer gewohnten Lernumgebung Stud.IP der Universität Göttingen zu- 
gänglich. Im Sinne von Open Science stehen alle Kurse zusätzlich für Nichtangehörige der Universität Göttingen über die Plattform OpenILIAS als OER zur Verfügung. Aufgrund der Verwendung fremder Materialien müssen die Kurse insgesamt unter der etwas einschränkenden, aber immer noch freien Lizenz CC BY-SA ${ }^{20}$ veröffentlicht werden. Selbst erstellte Grafiken und andere Materialien werden unter die möglichst freie Lizenz CCO gestellt. Diese Lizenzierungen ermöglichen es Lehrenden, die Kurse bzw. die Materialien kostenlos für eigene Lehrprojekte zu nutzen, d.h., die Kursinhalte zu verbreiten, zu bearbeiten und zu ergänzen. Sie können ganze Kurse oder einzelne Module kostenfrei verwenden und in ihre Lehrveranstaltungen einbinden bzw. an ihre eigenen, fachspezifischen Inhalte anpassen.

\section{Fazit und Ausblick}

Die Kurse werden insgesamt gut angenommen: Bis September 2020 haben sich mehr als 1300 Studierende in Stud.IP für die Kurse angemeldet. Hinzu kommen die Lernenden auf der freien Plattform OpenILIAS. Die Nutzung ist hier allerdings anonymisiert, damit das Angebot möglichst niedrigschwellig ist. Aus diesem Grund lassen sich keine validen Nutzungszahlen ermitteln, es ist aber davon auszugehen, dass die Zahlen hier ähnlich sind.

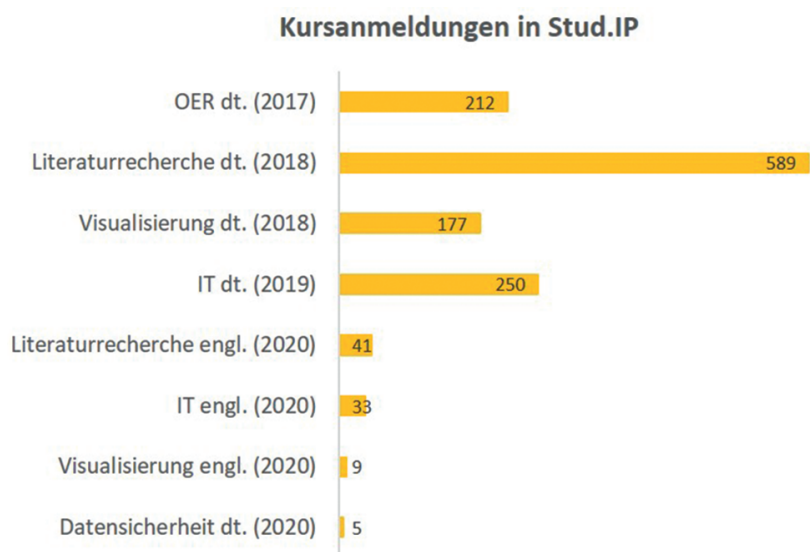

Abb. 4: Nutzung

Die Kurse wurden bis 2019 nur sehr punktuell beworben, da der Fokus auf der Erstellung von Inhalten lag. Ab dem SoSe 2019 wurde mit der Öffentlichkeitsreferentin des Projekts Campus $Q^{\text {PLUS }}$ (Franziska Peukert) und der Medienkoordinatorin für Studierende der Abteilung Studium und
Lehre (Sarvin Navidi) eine Strategie für die Bewerbung aller Kurse entwickelt. Dazu wurden Postkartentaschen entworfen und von einer Grafikerin ausgestaltet. Basierend auf diesem Design und einem Icon als Wiedererkennungseffekt wurden weitere Werbematerialien wie z.B. Plakate und Online-Werbung erstellt. Außerdem wurden besonders eingängige Tipps als kurze Videos umgesetzt, die über die Monitore auf dem Campus sowie über Instagram verbreitet werden. Zu guter Letzt wurde die Webseite des Projekts überarbeitet und stärker auf die Zielgruppe Studierende ausgerichtet.

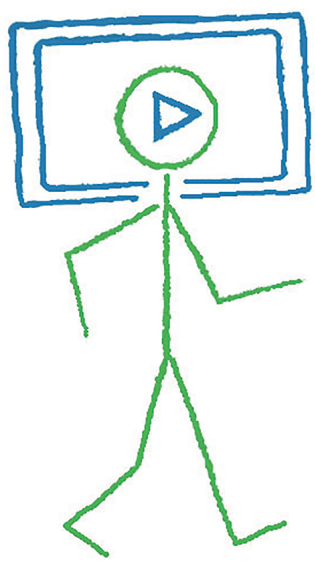

Abb. 5: Laufender Kurs

Mit den Selbstlernkursen waren die Universität Göttingen und insbesondere die SUB Göttingen gut auf die Durchführung eines digitalen Semesters ohne Präsenzlehre vorbereitet. Auf die Erfahrungen aus dem Projekt konnte schnell zurückgegriffen werden, so dass andere Lehrenden auf einzelne Elemente (z.B. H5P Quiz) oder ganze Kurse (z.B. Literaturrecherche) aufbauend eigene OnlineKurse erstellen konnten.

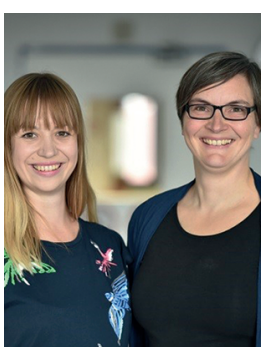

Silvia Czerwinski

Technische Informationsbibliothek (TIB)

Königsworther Platz 1 B

D-30167 Hannover

silvia.czerwinski@tib.eu

Tatyana Tasche

Georg-August-Universität Göttingen

Studium und Lehre

Platz der Göttinger Sieben 5

D-37073 Göttingen

tatyana.tasche@uni-goettingen.de

20 https://creativecommons.org/licenses/by-sa/4.0/deed.de. 